\title{
Even-order differential equation with continuous delay: nonexistence criteria of Kneser solutions
}

\author{
Ali Muhib ${ }^{1,2^{*}}$ (D) M. Motawi Khashan ${ }^{3}$ and Osama Moaaz
}

\section{"Correspondence:}

muhib39@yahoo.com

'Department of Mathematics,

Faculty of Science, Mansoura

University, 35516 Mansoura, Egypt

${ }^{2}$ Department of Mathematics,

Faculty of Education-Al-Nadirah, Ibb University, Ibb, Yemen

Full list of author information is available at the end of the article

\begin{abstract}
In this paper, we study even-order DEs where we deduce new conditions for nonexistence Kneser solutions for this type of DEs. Based on the nonexistence criteria of Kneser solutions, we establish the criteria for oscillation that take into account the effect of the delay argument, where to our knowledge all the previous results neglected the effect of the delay argument, so our results improve the previous results. The effectiveness of our new criteria is illustrated by examples.
\end{abstract}

MSC: $34 \mathrm{C} 10 ; 34 \mathrm{~K} 11$

Keywords: Even-order differential equations; Oscillatory solutions; Kneser solutions

\section{Introduction}

There is no doubt that the theory of oscillation of DEs is a fertile study area and has attracted the attention of many researchers recently. This is due to the existence of many important applications of this theory in various fields of applied science, see [18, 19]. In the last decade, it is easy to notice the new research movement that aims to improve and develop the criteria for oscillations of DEs of different orders, see [3-5] and [9-17].

In detail, we consider the even-order delay DE of the form

$$
\left(r \cdot\left(y^{(n-1)}\right)^{\gamma}\right)^{\prime}(\varsigma)+A\left[q \cdot(y \circ g)^{\gamma} ; a, b\right](\varsigma)=0, \quad \varsigma \geq \varsigma_{0},
$$

where $n \geq 4$ is an even natural number, $\gamma$ is quotient of odd positive integers, and $A[f ; a, b](\varsigma):=\int_{a}^{b} f(\varsigma, \varrho) \mathrm{d} \varrho$. Our study is under the following conditions:

$(\Omega 1) r \in C^{1}\left(I_{0},(0, \infty)\right), r^{\prime}(\varsigma) \geq 0, \int_{\varsigma_{0}}^{\infty} r^{-1 / \gamma}(\xi) \mathrm{d} \xi<\infty$, and $I_{\vartheta}:=\left[\varsigma_{\vartheta}, \infty\right)$;

$(\Omega 2) q \in C\left(I_{0} \times[a, b],[0, \infty)\right)$ and $q$ is not zero on any half line $[T, \infty) \times[a, b]$ for all $T \geq \varsigma_{0}$

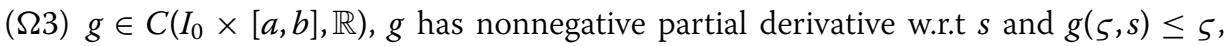
$\lim _{\zeta \rightarrow \infty} g(\varsigma, s)=\infty$ for all $s \in[a, b]$.

A solution of (1.1) means a function $y \in C^{(n-1)}\left(I_{y}, \mathbb{R}\right), \varsigma_{y} \geq \varsigma_{0}$, which satisfies the property $r \cdot\left(y^{(n-1)}\right)^{\gamma} \in C^{1}\left(I_{y}, \mathbb{R}\right)$; moreover, it satisfies (1.1) on $I_{y}$. We consider only the proper solutions $y$ of (1.1), that is, $y$ is not identically zero eventually.

(c) The Author(s) 2021. This article is licensed under a Creative Commons Attribution 4.0 International License, which permits use, sharing, adaptation, distribution and reproduction in any medium or format, as long as you give appropriate credit to the original author(s) and the source, provide a link to the Creative Commons licence, and indicate if changes were made. The images or other third party material in this article are included in the article's Creative Commons licence, unless indicated otherwise in a credit line to the material. If material is not included in the article's Creative Commons licence and your intended use is not permitted by statutory regulation or exceeds the permitted use, you will need to obtain permission directly from the copyright holder. To view a copy of this licence, visit http://creativecommons.org/licenses/by/4.0/. 
Definition 1.1 A solution $y$ of (1.1) is called a Kneser solution if there exists $\zeta_{*} \in I_{0}$ such that $y(\varsigma) y^{\prime}(\varsigma)<0$ for all $\varsigma \geq \varsigma_{*}$. (The set of all eventually positive Kneser solutions of (1.1) is denoted by $\mathbf{K}$.)

Definition 1.2 A solution $y$ of (1.1) is said to be nonoscillatory if it is positive or negative, ultimately; otherwise, it is said to be oscillatory. The equation itself is termed oscillatory if all its solutions oscillate.

Next, let us briefly review a number of closely related results which motivated the present study.

Li and Rogovchenko [9] were concerned with the asymptotic behavior of a class of higher-order sublinear Emden-Fowler delay DEs

$$
\left(r(\varsigma) y^{(n-1)}(\varsigma)\right)^{\prime}+q(\varsigma) y^{\beta}(\tau(\varsigma))=0
$$

where $0<\beta<1$ is a ratio of odd natural numbers and $\tau(\varsigma)<\varsigma$. They established two tests for the asymptotic behavior of solutions to the above equations. Moreover, they improved the theorems reported by Li and Rogovchenko [8] and Zhang et al. [20, 22].

Moaaz and Muhib [17] and Zhang et al. [21] presented criteria for oscillation of solutions of the DE

$$
\left(r(\varsigma)\left(y^{\prime \prime \prime}(\varsigma)\right)^{\gamma}\right)^{\prime}+f(\varsigma, y(\sigma(\varsigma)))=0,
$$

where $f(\varsigma, y) \geq h(\varsigma) y^{\beta}, \gamma, \beta$ are quotients of odd natural numbers and $\sigma(\varsigma)<\varsigma$. Results in [17] are an improvement on some of the results obtained in Zhang et al. [2].

Recently, Moaaz et al. [14] studied the oscillation and the asymptotic behavior of solutions of the DE

$$
\left(r(\varsigma)\left(y^{\prime \prime \prime}(\varsigma)\right)^{\gamma}\right)^{\prime}+q(\varsigma) y^{\beta}(\sigma(\varsigma))=0
$$

with the middle term

$$
\left(r(\varsigma)\left(y^{\prime \prime \prime}(\varsigma)\right)^{\gamma}\right)^{\prime}+p(\varsigma)\left(y^{\prime \prime \prime}(\varsigma)\right)^{\gamma}+q(\varsigma) y^{\beta}(\sigma(\varsigma))=0
$$

under the condition

$$
\int_{s_{0}}^{\infty} \frac{1}{r^{1 / \gamma}(s)} \mathrm{d} s=\infty
$$

and the condition

$$
\int_{s_{0}}^{\infty}\left[\frac{1}{r(s)} \exp \left(-\int_{s_{0}}^{s} \frac{p(\xi)}{r(\xi)} d \xi\right)\right]^{1 / \gamma} d s=\infty
$$

where $r^{\prime}(\varsigma)+p(\varsigma) \geq 0$.

In the paper, we are working on finding new criteria for oscillation of solutions of a class of even-order DEs in a noncanonical case. The paper is organized as follows. In Sect. 2, 
we present new conditions for the nonexistence of Kneser solutions of nonlinear evenorder DEs with continuous delay arguments. In Sect. 3, we are taking advantage of the new nonexistence criteria of Kneser solutions to create better criteria that ensure all solutions of (1.1) are oscillatory. In Sect. 4, we illustrate the effectiveness of our new criteria with examples.

Now, we provide the lemmas that will be needed during the results.

Lemma 1.1 ([1, Lemma 2.2.3]) Assume that $\varpi \in C^{n}\left(I_{0}, \mathbb{R}^{+}\right)$and $\varpi^{(n)}$ are of fixed sign and not identically zero on a subray of $I_{0}$. Furthermore, suppose that there exists $\varsigma_{1} \in I_{0}$ such that $\varpi^{(n-1)} \varpi^{(n)} \leq 0$ for $\varsigma \in I_{1}$. If $\lim _{\varsigma \rightarrow \infty} \varpi(\varsigma) \neq 0$, then there exists $\varsigma_{\lambda} \in I_{1}$ such that

$$
\varpi \geq \frac{\lambda}{(n-1) !} \varsigma^{n-1}\left|\varpi^{(n-1)}\right|
$$

for every $\lambda \in(0,1)$ and $\varsigma \in I_{\lambda}$.

Lemma 1.2 Let $\varpi(\xi)=D \xi-M(\xi-N)^{(\gamma+1) / \gamma}$, where $M>0, D$ and $N$ are constants. Then the maximum value of $\varpi$ on $R$ at $\xi^{*}=N+(\gamma D /((\gamma+1) M))^{\gamma}$ is

$$
\max _{\xi \in R} \varpi(\xi)=\varpi\left(\xi^{*}\right)=D N+\frac{\gamma^{\gamma}}{(\gamma+1)^{(\gamma+1)}} \frac{D^{\gamma+1}}{M^{\gamma}} .
$$

\section{Nonexistence of Kneser solutions}

Firstly, we define the notations $\delta_{0}(\varsigma):=\int_{\varsigma}^{\infty} r^{-1 / \gamma}(\xi) \mathrm{d} \xi$ and $\delta_{m}(\varsigma):=\int_{\varsigma}^{\infty} \delta_{m-1}(\xi) \mathrm{d} \xi$ for $m=$ $1,2, \ldots, n-2$. The following lemma is an adaptation of Lemma 1.1 in [6] based on $n$ even.

Lemma 2.1 If $y$ is an eventually positive solution of (1.1), then $\left(r \cdot y^{(n-1)}\right)^{\prime} \leq 0$, and one of the following cases holds for 5 large enough:

(1) $y^{\prime}(\varsigma)>0, y^{(n-1)}(\varsigma)>0$ and $y^{(n)}(\varsigma)<0$;

(2) $y^{\prime}(\varsigma)>0, y^{(n-2)}(\varsigma)>0$ and $y^{(n-1)}(\varsigma)<0$;

(3) $(-1)^{k} y^{(k)}(\varsigma)>0$ for $k=1,2, \ldots, n-1$.

Remark 2.1 Based on the definition of the class $\mathbf{K}$, we note that $y \in \mathbf{K}$ if and only if $y$ satisfies case (3).

Lemma 2.2 Assume that $y \in \mathbf{K}$. Then y converges to zero if

$$
\int_{S_{0}}^{\infty}\left(\frac{1}{r(\xi)} \int_{\varsigma_{0}}^{\xi} A[q ; a, b](v) \mathrm{d} v\right)^{1 / \gamma} \mathrm{d} \xi=\infty
$$

Proof Based on the belonging of $y$ to $\mathbf{K}$, we note that $y$ is a positive decreasing function, and so $\lim _{\varsigma \rightarrow \infty} y(\varsigma)=\epsilon \geq 0$. Assuming the opposite of that, it is required that $\epsilon>0$. Then there exists $\varsigma_{1} \in I_{0}$ such that $y(\varsigma)>\epsilon$ for all $\varsigma \geq \varsigma_{1}$. Thus, from ( $\left.\Omega 3\right)$, there exists $\varsigma_{2} \geq \varsigma_{1}$ such that $(y \circ g)(\varsigma)>\epsilon$ for $\varsigma \geq \varsigma_{2}$. From (1.1), we arrive at

$$
\left(r \cdot\left(y^{(n-1)}\right)^{\gamma}\right)^{\prime}(\varsigma) \leq-\epsilon^{\gamma} A[q ; a, b](\varsigma) \text { for } \varsigma \geq \varsigma_{2}
$$


Integrating the above inequality from $\varsigma_{2}$ to $\varsigma$, we get

$$
\left(r \cdot\left(y^{(n-1)}\right)^{\gamma}\right)(\varsigma) \leq\left(r \cdot\left(y^{(n-1)}\right)^{\gamma}\right)\left(\varsigma_{2}\right)-\epsilon^{\gamma} \int_{\varsigma_{2}}^{\varsigma} A[q ; a, b](v) \mathrm{d} v,
$$

that is,

$$
y^{(n-1)}(\varsigma) \leq-\epsilon\left(\frac{1}{r(\varsigma)} \int_{\varsigma_{2}}^{\varsigma} A[q ; a, b](v) \mathrm{d} v\right)^{1 / \gamma}
$$

Integrating the last inequality from $\varsigma_{2}$ to $\zeta$, we obtain

$$
y^{(n-2)}(\varsigma) \leq y^{(n-2)}\left(\varsigma_{2}\right)-\epsilon \int_{\varsigma 2}^{\varsigma}\left(\frac{1}{r(\xi)} \int_{\varsigma_{2}}^{\xi} A[q ; a, b](v) \mathrm{d} v\right)^{1 / \gamma} \mathrm{d} \xi
$$

Taking $\lim _{\varsigma \rightarrow \infty}$ and assumption (2.1) into account, we get that $y^{(n-2)}(\varsigma) \rightarrow-\infty$ as $\varsigma \rightarrow \infty$, which is a contradiction. Thus, $\epsilon=0$. This completes the proof.

Lemma 2.3 Assume that (2.1) holds. If $y \in \mathbf{K}$, then

$$
-\left(r \cdot\left(y^{(n-1)}\right)^{\gamma}\right)(\varsigma) \geq \int_{\varsigma 0}^{\varsigma} A\left[q \cdot(y \circ g)^{\gamma} ; a, b\right](\xi) \mathrm{d} \xi
$$

and

$$
(-1)^{(k)} y^{(k)}(\varsigma) \geq-\left(r \cdot\left(y^{(n-1)}\right)^{\gamma}\right)^{1 / \gamma}(\varsigma) \delta_{n-2-k}(\varsigma)
$$

for $k=0,1, \ldots, n-2$.

Proof Assume that $y \in \mathbf{K}$ on $\left[\varsigma_{1}, \infty\right)$. Integrating (1.1) from $\varsigma_{1}$ to $\varsigma$ and using that fact that $y^{\prime}(\varsigma)<0$, we obtain

$$
\begin{aligned}
-\left(r \cdot\left(y^{(n-1)}\right)^{\gamma}\right)(\varsigma) \geq & -\left(r \cdot\left(y^{(n-1)}\right)^{\gamma}\right)\left(\varsigma_{1}\right)+\int_{\varsigma 1}^{\varsigma} A\left[q \cdot(y \circ g)^{\gamma} ; a, b\right](\xi) \mathrm{d} \xi \\
\geq & -\left(r \cdot\left(y^{(n-1)}\right)^{\gamma}\right)\left(\varsigma_{1}\right)+\int_{\varsigma_{0}}^{\varsigma} A\left[q \cdot(y \circ g)^{\gamma} ; a, b\right](\xi) \mathrm{d} \xi \\
& -\int_{\varsigma_{0}}^{\varsigma_{1}} A\left[q \cdot(y \circ g)^{\gamma} ; a, b\right](\xi) \mathrm{d} \xi
\end{aligned}
$$

for all $\varsigma \in I_{1}$. It follows from Lemma 2.2 that $y$ converges to zero. Then there is $\varsigma_{2} \in I_{1}$ such that, for $\varsigma \geq \varsigma_{2}$,

$$
\left(r \cdot\left(y^{(n-1)}\right)^{\gamma}\right)\left(\varsigma_{1}\right)+\int_{\varsigma_{0}}^{\varsigma_{1}} A\left[q \cdot(y \circ g)^{\gamma} ; a, b\right](\xi) \mathrm{d} \xi<0,
$$

which with (2.4) gives

$$
-\left(r \cdot\left(y^{(n-1)}\right)^{\gamma}\right)(\varsigma) \geq \int_{\varsigma 0}^{\varsigma} A\left[q \cdot(y \circ g)^{\gamma} ; a, b\right](\xi) \mathrm{d} \xi .
$$


Next, by using the fact that $\left(r^{1 / \gamma} \cdot y^{(n-1)}\right)^{\prime} \leq 0$, we see that

$$
\begin{aligned}
y^{(n-2)}(g(\varsigma, s)) & \geq y^{(n-2)}(\varsigma) \geq \int_{\varsigma}^{\infty} \frac{1}{r^{1 / \gamma}(\varrho)}\left(-r^{1 / \gamma}(\varrho) y^{(n-1)}(\varrho)\right) \mathrm{d} \varrho \\
& \geq-\left(r \cdot\left(y^{(n-1)}\right)^{\gamma}\right)^{1 / \gamma}(\varsigma) \delta_{0}(\varsigma) .
\end{aligned}
$$

Integrating (2.5) from $\varsigma$ to $\infty$ and taking the monotonicity of $y^{(n-3)}(\varsigma)$ into account, we find

$$
-y^{(n-3)}(\varsigma) \geq-\left(r \cdot\left(y^{(n-1)}\right)^{\gamma}\right)^{1 / \gamma}(\varsigma) \delta_{1}(\varsigma)
$$

Integrating again from $\varsigma$ to $\infty$, we obtain

$$
y^{(n-4)}(\varsigma) \geq-\left(r \cdot\left(y^{(n-1)}\right)^{\gamma}\right)^{1 / \gamma}(\varsigma) \delta_{2}(\varsigma)
$$

Going forward along the same method, we get

$$
(-1)^{(k)} y^{(k)}(\varsigma) \geq-\left(r \cdot\left(y^{(n-1)}\right)^{\gamma}\right)^{1 / \gamma}(\varsigma) \delta_{n-2-k}(\varsigma)
$$

for $k=0,1, \ldots, n-2$. This completes the proof.

Theorem 2.2 Assume that (2.1) holds. If

$$
\eta:=\underset{\varsigma \rightarrow \infty}{\limsup } \delta_{n-2}(\varsigma)\left(\int_{\varsigma_{0}}^{\varsigma} A[q ; a, b](\xi) \mathrm{d} \xi\right)^{1 / \gamma}>1
$$

then $\mathbf{K}=\varnothing$.

Proof Suppose to the contrary that $y \in \mathbf{K}$ on $\left[\varsigma_{1}, \infty\right)$. From Lemma 2.3, we obtain (2.2) and (2.3) hold. Since $g$ is delay w.s.t $\varsigma$, we get $y \circ g \geq y$ for $\varsigma \geq \varsigma_{2}$ and $s \in[a, b]$. Thus, (2.2) becomes

$$
-\left(r \cdot\left(y^{(n-1)}\right)^{\gamma}\right)(\varsigma) \geq y^{\gamma}(\varsigma) \int_{\varsigma 0}^{\varsigma} A[q ; a, b](\xi) \mathrm{d} \xi
$$

which with ((2.3), $k=0)$ gives

$$
-\left(r \cdot\left(y^{(n-1)}\right)^{\gamma}\right)(\varsigma) \geq-\left(r \cdot\left(y^{(n-1)}\right)^{\gamma}\right)(\varsigma) \delta_{n-2}^{\gamma}(\varsigma) \int_{\varsigma 0}^{\varsigma} A[q ; a, b](\xi) \mathrm{d} \xi
$$

or equivalently,

$$
1 \geq \delta_{n-2}^{\gamma}(\varsigma) \int_{\varsigma_{0}}^{\varsigma} A[q ; a, b](\xi) \mathrm{d} \xi
$$

Taking the limsup on both sides of the inequality, we arrive at contradiction with (2.6). This completes the proof.

For the next results, we introduce the following additional condition: 
( $\Omega)$ There is a constant $h>1 \mathrm{t}$ such that $\frac{\delta_{n-2}(g(\varsigma, s))}{\delta_{n-2}(\varsigma)} \geq h$ for $\varsigma \geq \varsigma_{0}$ and $s \in[a, b]$.

Lemma 2.4 Assume that $y \in \mathbf{K},(2.1)$ hold and $\eta$ is defined as in (2.6). Then there exists $\varsigma_{\varepsilon} \geq \varsigma_{1}$ such that

$$
\frac{\mathrm{d}}{\mathrm{d} \varsigma}\left(\frac{y(\varsigma)}{\delta_{n-2}^{\eta-\varepsilon}(\varsigma)}\right) \leq 0
$$

for any $\varepsilon>0$ and $\varsigma \geq \varsigma_{\varepsilon}$. Moreover, if $(\Omega)$ holds, then

$$
y(g(\varsigma, s)) \geq h^{\eta-\varepsilon} y(\varsigma) \text { for } \varsigma \geq \varsigma_{\varepsilon} \text { and } s \in[a, b] .
$$

Proof Assume that $y \in \mathbf{K}$ on $I_{1}$. From Lemma 2.3, we obtain (2.2) and (2.3) hold. It follows from (2.2) and the fact that $g(\varsigma, s) \leq \varsigma$ that

$$
-\left(r \cdot\left(y^{(n-1)}\right)^{\gamma}\right)(\varsigma) \geq y^{\gamma}(\varsigma) \int_{\varsigma 0}^{\varsigma} A[q ; a, b](\xi) \mathrm{d} \xi
$$

From the definition of $\eta$ in Theorem 2.2, there exists $\varsigma_{2} \geq \varsigma_{1}$ such that

$$
\delta_{n-2}\left(\int_{\zeta_{0}}^{\varsigma} A[q ; a, b](\xi) \mathrm{d} \xi\right)^{1 / \gamma}>\eta_{*}:=\eta-\varepsilon
$$

for all $\varepsilon>0$ and $\varsigma \geq \varsigma_{2}$. Hence, from ((2.3), $\left.k=1\right)$, we have

$$
\frac{\mathrm{d}}{\mathrm{d} \varsigma}\left(\frac{y(\varsigma)}{\delta_{n-2}^{\eta_{*}}(\varsigma)}\right) \leq \frac{\delta_{n-2}^{\eta_{*}}(\varsigma) r^{1 / \gamma}(\varsigma) y^{(n-1)}(\varsigma) \delta_{n-3}(\varsigma)}{\delta_{n-2}^{2 \eta_{*}}(\varsigma)}+\frac{y(\varsigma) \eta_{*} \delta_{n-2}^{\eta_{*}-1}(\varsigma) \delta_{n-3}(\varsigma)}{\delta_{n-2}^{2 \eta_{*}}(\varsigma)}
$$

which with (2.8) gives

$$
\begin{aligned}
\frac{\mathrm{d}}{\mathrm{d} \varsigma}\left(\frac{y(\varsigma)}{\delta_{n-2}^{\eta_{*}}(\varsigma)}\right) \leq & \frac{-y(\varsigma) \delta_{n-2}^{\eta_{*}}(\varsigma) \delta_{n-3}(\varsigma)}{\delta_{n-2}^{2 \eta_{*}}(\varsigma)}\left(\int_{\varsigma 0}^{\varsigma} A[q ; a, b](\xi) \mathrm{d} \xi\right)^{1 / \gamma} \\
& +\frac{y(\varsigma) \eta_{*} \delta_{n-2}^{\eta_{*}-1}(\varsigma) \delta_{n-3}(\varsigma)}{\delta_{n-2}^{2 \eta_{*}}(\varsigma)} \\
\leq & \frac{y(\varsigma) \delta_{n-3}(\varsigma)}{\delta_{n-2}^{\eta_{*+1}}(\varsigma)}\left(\eta_{*}-\delta_{n-2}(\varsigma)\left(\int_{\varsigma_{0}}^{\varsigma} A[q ; a, b](\xi) \mathrm{d} \xi\right)^{1 / \gamma}\right) \leq 0 .
\end{aligned}
$$

Using this fact, one can easily see that

$$
y(g(\varsigma, s)) \geq y(\varsigma)\left(\frac{\delta_{n-2}(g(\varsigma, s))}{\delta_{n-2}(\varsigma)}\right)^{\eta_{*}} \geq h^{\eta_{*}} y(\varsigma)
$$

This completes the proof.

Theorem 2.3 Assume that $(\Omega),(2.1)$ hold and $\eta$ is defined as in (2.6). If

$$
h^{\eta} \eta>1
$$

then $\mathbf{K}=\varnothing$. 
Proof Suppose to the contrary that $y \in \mathbf{K}$ on $I_{1}$. From Lemma 2.3 and 2.4, we obtain that (2.2), (2.3), and (2.7) hold. Combining (2.2) and (2.7), we obtain

$$
-\left(r \cdot\left(y^{(n-1)}\right)^{\gamma}\right)(\varsigma) \geq y^{\gamma}(\varsigma) h^{\gamma \eta_{*}} \int_{\varsigma 0}^{\varsigma} A[q ; a, b](\xi) \mathrm{d} \xi
$$

for all $\varepsilon>0$ and $\varsigma \geq \varsigma_{1}$. Using $((2.3), k=0)$, we have

$$
-\left(r \cdot\left(y^{(n-1)}\right)^{\gamma}\right)(\varsigma) \geq-\left(r \cdot\left(y^{(n-1)}\right)^{\gamma}\right)(\varsigma) \delta_{n-2}^{\gamma}(\varsigma) h^{\gamma \eta_{*}} \int_{\varsigma_{0}}^{\varsigma} A[q ; a, b](\xi) \mathrm{d} \xi
$$

Taking the limsup on both sides of the latter inequality, we obtain $h^{\eta} \eta \leq 1$. Then we obtain a contradiction with (2.9). This completes the proof.

Theorem 2.4 Assume that ( $\Omega$ ), (2.1) hold and $\eta$ is defined as in (2.6). If

$$
\limsup _{\varsigma \rightarrow \infty} \int_{\varsigma 0}^{\varsigma}\left(h^{\gamma \eta} \delta_{n-2}^{\gamma}(\varrho) A[q ; a, b](\varrho)-\frac{\gamma^{\gamma+1}}{(\gamma+1)^{\gamma+1}} \frac{\delta_{n-3}(\varrho)}{\delta_{n-2}(\varrho)}\right) \mathrm{d} \varrho=\infty
$$

then $\mathbf{K}=\varnothing$.

Proof Suppose to the contrary that $y \in \mathbf{K}$ on $I_{1}$. From Lemmas 2.3 and 2.4, we obtain that (2.2), (2.3), and (2.7) hold. Define the function

$$
\omega(\varsigma):=\frac{\left(r \cdot\left(y^{(n-1)}\right)^{\gamma}\right)(\varsigma)}{y^{\gamma}(\varsigma)}
$$

Differentiating $\omega(\varsigma)$, we get

$$
\omega^{\prime}(\varsigma)=\frac{\left(r(\varsigma)\left(y^{(n-1)}(\varsigma)\right)^{\gamma}\right)^{\prime}}{y^{\gamma}(\varsigma)}-\frac{\gamma r(\varsigma)\left(y^{(n-1)}(\varsigma)\right)^{\gamma} y^{\prime}(\varsigma)}{y^{\gamma+1}(\varsigma)}
$$

Using (1.1), ((2.3), $k=1)$, and (2.7), we arrive at

$$
\omega^{\prime}(\varsigma) \leq-h^{\gamma \eta_{*}} A[q ; a, b](\varsigma)-\gamma \delta_{n-3}(\varsigma) \omega^{(\gamma+1) / \gamma}(\varsigma)
$$

Multiplying (2.12) by $\delta_{n-2}^{\gamma}$ and integrating the resulting inequality from $\varsigma_{1}$ to $\varsigma$, we obtain

$$
\begin{aligned}
\delta_{n-2}^{\gamma}(\varsigma) \omega(\varsigma)-\delta_{n-2}^{\gamma}\left(\varsigma_{1}\right) \omega\left(\varsigma_{1}\right) \leq & -\int_{\varsigma 1}^{\varsigma} h^{\gamma \eta_{*}} \delta_{n-2}^{\gamma}(\varrho) A[q ; a, b](\varrho) \mathrm{d} \varrho \\
& -\int_{\varsigma 1}^{\varsigma} \gamma \delta_{n-2}^{\gamma-1}(\varrho) \delta_{n-3}(\varrho) \omega(\varrho) \mathrm{d} \varrho \\
& -\int_{\varsigma 1}^{\varsigma} \gamma \delta_{n-3}(\varrho) \delta_{n-2}^{\gamma}(\varrho) \omega^{(\gamma+1) / \gamma}(\varrho) \mathrm{d} \varrho .
\end{aligned}
$$

Using the inequality

$$
-B v+A v^{(\gamma+1) / \gamma} \geq-\frac{\gamma^{\gamma}}{(\gamma+1)^{\gamma+1}} \frac{B^{\gamma+1}}{A^{\gamma}}, \quad A, B>0
$$


with $A=\delta_{n-3}(\varrho) \delta_{n-2}^{\gamma}(\varrho), B=\delta_{n-2}^{\gamma-1}(\varrho) \delta_{n-3}(\varrho)$, and $v=-\omega(\varrho)$, we conclude that

$$
\begin{aligned}
& \int_{\varsigma 1}^{\varsigma}\left(h^{\gamma \eta_{*}} \delta_{n-2}^{\gamma}(\varrho) A[q ; a, b](\varrho)-\frac{\gamma^{\gamma+1}}{(\gamma+1)^{\gamma+1}} \frac{\delta_{n-3}(\varrho)}{\delta_{n-2}(\varrho)}\right) \mathrm{d} \varrho \\
& \quad \leq \delta_{n-2}^{\gamma}\left(\varsigma_{1}\right) \omega\left(\varsigma_{1}\right)-\delta_{n-2}^{\gamma}(\varsigma) \omega(\varsigma) .
\end{aligned}
$$

From ((2.3), $k=0)$, one can easily see that $-1 \leq \omega(\varsigma) \delta_{n-2}^{\gamma}(\varsigma)<0$, which with (2.13) gives

$$
\int_{\varsigma 1}^{\varsigma}\left(h^{\gamma \eta_{*}} \delta_{n-2}^{\gamma}(\varrho) A[q ; a, b](\varrho)-\frac{\gamma^{\gamma+1}}{(\gamma+1)^{\gamma+1}} \frac{\delta_{n-3}(\varrho)}{\delta_{n-2}(\varrho)}\right) \mathrm{d} \varrho<1+\delta_{n-2}^{\gamma}\left(\varsigma_{1}\right) \omega\left(\varsigma_{1}\right) .
$$

Taking the limsup on both sides of the latter inequality, we obtain a contradiction with (2.11). This completes the proof.

Theorem 2.5 Assume that $(\Omega)$ and (2.1) hold. If there exists a function $\rho \in C^{1}\left(I_{0},(0, \infty)\right)$ such that

$$
\limsup _{\varsigma \rightarrow \infty} \frac{\delta_{n-2}^{\gamma}(\varsigma)}{\rho(\varsigma)} \int_{\varsigma 1}^{\varsigma}\left(\rho(\zeta) h^{\gamma \eta} A[q ; a, b](\zeta)-\frac{\rho^{-\gamma}(\zeta)\left(\rho^{\prime}(\zeta)\right)^{\gamma+1}}{(\gamma+1)^{(\gamma+1)} \delta_{n-3}^{\gamma}(\zeta)}\right) \mathrm{d} \zeta>1
$$

then $\mathbf{K}=\varnothing$.

Proof Suppose to the contrary that $y \in \mathbf{K}$ on $I_{1}$. Using Lemmas 2.3 and 2.4, we obtain that (2.2), (2.3), and (2.7) hold. From ((2.3), $k=0)$, we obtain

$$
\frac{\left(r \cdot\left(y^{(n-1)}\right)^{\gamma}\right)(\varsigma)}{y^{\gamma}(\varsigma)} \geq-\frac{1}{\delta_{n-2}^{\gamma}(\varsigma)}
$$

Thus, if we define a generalized Riccati substitution as

$$
w(\varsigma):=\rho\left(\frac{r \cdot\left(y^{(n-1)}\right)^{\gamma}}{y^{\gamma}}+\frac{1}{\delta_{n-2}^{\gamma}}\right)
$$

then $w(\varsigma)>0$ for all $\varsigma \geq \varsigma_{1}$. Differentiating $\omega$, we have

$$
\begin{aligned}
w^{\prime}(\varsigma)= & \frac{\rho^{\prime}(\varsigma)}{\rho(\varsigma)} w(\varsigma)+\rho(\varsigma) \frac{\left(r \cdot\left(y^{(n-1)}\right)^{\gamma}\right)^{\prime}(\varsigma)}{y^{\gamma}(\varsigma)} \\
& -\gamma \rho(\varsigma) \frac{\left(r \cdot\left(y^{(n-1)}\right)^{\gamma}\right)(\varsigma)}{y^{\gamma+1}(\varsigma)} y^{\prime}(\varsigma) \\
& -\frac{\gamma \rho(\varsigma) \delta_{n-2}^{\prime}(\varsigma)}{\delta_{n-2}^{\gamma+1}(\varsigma)} .
\end{aligned}
$$

From (1.1), we see that

$$
\left(r(\varsigma)\left(y^{(n-1)}(\varsigma)\right)^{\gamma}\right)^{\prime}=-A\left[q \cdot(y \circ g)^{\gamma} ; a, b\right](\varsigma) \leq 0 .
$$


Using ((2.3), $k=1)$ and (2.18), (2.17) becomes

$$
\begin{aligned}
w^{\prime}(\varsigma) \leq & \frac{\rho^{\prime}(\varsigma)}{\rho(\varsigma)} w(\varsigma)-\rho(\varsigma) \frac{A\left[q \cdot(y \circ g)^{\gamma} ; a, b\right](\varsigma)}{y^{\gamma}(\varsigma)} \\
& -\gamma \rho(\varsigma) r(\varsigma)\left(\frac{y^{(n-1)}(\varsigma)}{y(\varsigma)}\right)^{\gamma+1} r^{1 / \gamma}(\varsigma) \delta_{n-3}(\varsigma)+\frac{\gamma \rho(\varsigma) \delta_{n-3}(\varsigma)}{\delta_{n-2}^{\gamma+1}(\varsigma)} .
\end{aligned}
$$

Thus, from (2.7), (2.19) yields

$$
\begin{aligned}
w^{\prime}(\varsigma) \leq & \frac{\rho^{\prime}(\varsigma)}{\rho(\varsigma)} w(\varsigma)-\rho(\varsigma) h^{\gamma(\eta-\varepsilon)} A[q ; a, b](\varsigma)+\frac{\gamma \rho(\varsigma) \delta_{n-3}(\varsigma)}{\delta_{n-2}^{\gamma+1}(\varsigma)} \\
& -\gamma \rho(\varsigma) r(\varsigma)\left(\frac{y^{(n-1)}(\varsigma)}{y(\varsigma)}\right)^{\gamma+1} r^{1 / \gamma}(\varsigma) \delta_{n-3}(\varsigma) .
\end{aligned}
$$

Therefore, from the definition of $w$, we get

$$
\begin{aligned}
w^{\prime}(\varsigma) \leq & -\rho(\varsigma) h^{\gamma(\eta-\varepsilon)} A[q ; a, b](\varsigma)+\frac{\gamma \rho(\varsigma) \delta_{n-3}(\varsigma)}{\delta_{n-2}^{\gamma+1}(\varsigma)} \\
& +\frac{\rho^{\prime}(\varsigma)}{\rho(\varsigma)} w(\varsigma)-\gamma \frac{\delta_{n-3}(\varsigma)}{\rho^{1 / \gamma}(\varsigma)}\left(w(\varsigma)-\frac{\rho(\varsigma)}{\delta_{n-2}^{\gamma}(\varsigma)}\right)^{1+1 / \gamma} .
\end{aligned}
$$

Using inequality (1.2) with

$$
D:=\frac{\rho^{\prime}(\varsigma)}{\rho(\varsigma)}, \quad M:=\gamma \frac{\delta_{n-3}(\varsigma)}{\rho^{1 / \gamma}(\varsigma)}, \quad N:=\frac{\rho(\varsigma)}{\delta_{n-2}^{\gamma}(\varsigma)}
$$

and $\xi:=w$, we obtain

$$
\frac{\rho^{\prime}(\varsigma)}{\rho(\varsigma)} w(\varsigma) \leq \gamma \frac{\delta_{n-3}(\varsigma)}{\rho^{1 / \gamma}(\varsigma)}\left(w(\varsigma)-\frac{\rho(\varsigma)}{\delta_{n-2}^{\gamma}(\varsigma)}\right)^{1+1 / \gamma}+\frac{\rho^{\prime}(\varsigma)}{\delta_{n-2}^{\gamma}(\varsigma)}+\frac{\rho^{-\gamma}(\varsigma)\left(\rho^{\prime}(\varsigma)\right)^{\gamma+1}}{(\gamma+1)^{(\gamma+1)} \delta_{n-3}^{\gamma}(\varsigma)}
$$

which, with (2.20), gives

$$
\begin{aligned}
w^{\prime}(\varsigma) \leq & -\rho(\varsigma) h^{\gamma(\eta-\varepsilon)} A[q ; a, b](\varsigma) \\
& +\frac{\rho^{-\gamma}(\varsigma)\left(\rho^{\prime}(\varsigma)\right)^{\gamma+1}}{(\gamma+1)^{(\gamma+1)} \delta_{n-3}^{\gamma}(\varsigma)}+\frac{\rho^{\prime}(\varsigma)}{\delta_{n-2}^{\gamma}(\varsigma)}+\frac{\gamma \rho(\varsigma) \delta_{n-3}(\varsigma)}{\delta_{n-2}^{\gamma+1}(\varsigma)}
\end{aligned}
$$

or

$$
w^{\prime}(\varsigma) \leq-\rho(\varsigma) h^{\gamma(\eta-\varepsilon)} A[q ; a, b](\varsigma)+\frac{\rho^{-\gamma}(\varsigma)\left(\rho^{\prime}(\varsigma)\right)^{\gamma+1}}{(\gamma+1)^{(\gamma+1)} \delta_{n-3}^{\gamma}(\varsigma)}+\frac{\mathrm{d}}{\mathrm{d} \varsigma}\left(\frac{\rho(\varsigma)}{\delta_{n-2}^{\gamma}(\varsigma)}\right) .
$$

Integrating this inequality from $\varsigma_{1}$ to $\varsigma$, we arrive at

$$
\begin{aligned}
w(\varsigma)-w\left(\varsigma_{1}\right) \leq & -\int_{\varsigma_{1}}^{\varsigma}\left(\rho(\zeta) h^{\gamma(\eta-\varepsilon)} A[q ; a, b](\zeta)-\frac{\rho^{-\gamma}(\zeta)\left(\rho^{\prime}(\zeta)\right)^{\gamma+1}}{(\gamma+1)^{(\gamma+1)} \delta_{n-3}^{\gamma}(\zeta)}\right) \mathrm{d} \zeta \\
& +\frac{\rho(\varsigma)}{\delta_{n-2}^{\gamma}(\varsigma)}-\frac{\rho\left(\varsigma_{1}\right)}{\delta_{n-2}^{\gamma}\left(\varsigma_{1}\right)} .
\end{aligned}
$$


From (2.16), we are led to

$$
\begin{aligned}
& \int_{\zeta 1}^{\varsigma}\left(\rho(\zeta) h^{\gamma(\eta-\varepsilon)} A[q ; a, b](\zeta)-\frac{\rho^{-\gamma}(\zeta)\left(\rho^{\prime}(\zeta)\right)^{\gamma+1}}{(\gamma+1)^{(\gamma+1)} \delta_{n-3}^{\gamma}(\zeta)}\right) \mathrm{d} \zeta \\
& \quad \leq-\rho(\varsigma) \frac{r(\varsigma)\left(y^{(n-1)}(\varsigma)\right)^{\gamma}}{y^{\gamma}(\varsigma)}+\rho\left(\varsigma_{1}\right) \frac{r\left(\varsigma_{1}\right)\left(y^{(n-1)}\left(\varsigma_{1}\right)\right)^{\gamma}}{y^{\gamma}\left(\varsigma_{1}\right)} \\
& \quad \leq-\rho(\varsigma) \frac{r(\varsigma)\left(y^{(n-1)}(\varsigma)\right)^{\gamma}}{y^{\gamma}(\varsigma)} .
\end{aligned}
$$

In view of (2.15), we get

$$
\int_{\varsigma 1}^{\varsigma}\left(\rho(\zeta) h^{\gamma(\eta-\varepsilon)} A[q ; a, b](\zeta)-\frac{\rho^{-\gamma}(\zeta)\left(\rho^{\prime}(\zeta)\right)^{\gamma+1}}{(\gamma+1)^{(\gamma+1)} \delta_{n-3}^{\gamma}(\zeta)}\right) \mathrm{d} \zeta \leq \frac{\rho(\varsigma)}{\delta_{n-2}^{\gamma}(\varsigma)}
$$

or

$$
\frac{\delta_{n-2}^{\gamma}(\varsigma)}{\rho(\varsigma)} \int_{\zeta 1}^{\zeta}\left(\rho(\zeta) h^{\gamma(\eta-\varepsilon)} A[q ; a, b](\zeta)-\frac{\rho^{-\gamma}(\zeta)\left(\rho^{\prime}(\zeta)\right)^{\gamma+1}}{(\gamma+1)^{(\gamma+1)} \delta_{n-3}^{\gamma}(\zeta)}\right) \mathrm{d} \zeta \leq 1
$$

Taking the limsup, we obtain a contradiction. This completes the proof.

Corollary 2.1 Assume that $(\Omega)$ and (2.1) hold. If one of the following conditions holds:

$$
\limsup _{\varsigma \rightarrow \infty} \delta_{n-2}^{\gamma}(\varsigma) \int_{\varsigma_{1}}^{\varsigma} h^{\gamma \eta} A[q ; a, b](\zeta) \mathrm{d} \zeta>1
$$

or

$$
\limsup _{\varsigma \rightarrow \infty} \delta_{n-2}^{\gamma-1}(\varsigma) \int_{\varsigma 1}^{\varsigma}\left(h^{\gamma \eta} \delta_{n-2}(\zeta) A[q ; a, b](\zeta)-\frac{1}{(\gamma+1)^{(\gamma+1)}} \frac{\delta_{n-3}(\zeta)}{\delta_{n-2}^{\gamma}(\zeta)}\right) \mathrm{d} \zeta>1,
$$

or

$$
\limsup _{\zeta \rightarrow \infty} \int_{\varsigma 1}^{\varsigma}\left(h^{\gamma \eta} \delta_{n-2}^{\gamma}(\zeta) A[q ; a, b](\zeta)-\frac{\gamma^{\gamma+1}}{(\gamma+1)^{(\gamma+1)}} \frac{\delta_{n-3}(\varsigma)}{\delta_{n-2}(\varsigma)}\right) \mathrm{d} \zeta>1
$$

then $\mathbf{K}=\varnothing$.

Proof By choosing $\rho(\varsigma)=1, \rho(\varsigma)=\delta_{2}(\varsigma)$, and $\rho(\varsigma)=\delta_{2}^{\gamma}(\varsigma)$, condition (2.14) in Theorem 2.5 becomes as $(2.21),(2.22)$, and (2.23), respectively.

\section{Oscillation criteria}

In this section, we are taking advantage of new nonexistence criteria of Kneser solutions to create better criteria that ensure all solutions of (1.1) are oscillatory.

Theorem 3.1 Assume that $(\Omega)$ and (2.1) hold and there exists a function $\rho \in C^{1}\left(I_{0},(0, \infty)\right)$ such that (2.14) holds. If the DE

$$
v^{\prime}(\varsigma)+\left(\frac{\lambda_{0} g^{n-1}(\varsigma, a)}{(n-1) !}\right)^{\gamma} \frac{A[q ; a, b](\varsigma)}{r(g(\varsigma, a))} v(g(\varsigma, a))=0
$$


is oscillatory for some $\lambda_{0} \in(0,1)$ and there exists a function $\theta \in C^{1}\left(I_{0},(0, \infty)\right)$ such that

$$
\limsup _{\varsigma \rightarrow \infty} \int_{\varsigma_{0}}^{\varsigma}\left(\Phi(\varrho)-\frac{r(\varrho) \theta(\varrho)}{(\gamma+1)^{\gamma+1}}\left(\frac{\theta^{\prime}(\varrho)}{\theta(\varrho)}+\frac{1+\gamma}{r^{1 / \gamma}(\varrho) \delta_{0}(\varrho)}\right)^{\gamma+1}\right) \mathrm{d} \varrho=\infty
$$

holds for some $\lambda_{1} \in(0,1)$, where

$$
\Phi:=\theta(\varsigma)\left(\frac{\lambda_{1} g^{n-2}(\varsigma, a)}{(n-2) !}\right)^{\gamma} A[q ; a, b](\varsigma)+(1-\gamma) \frac{\theta(\varsigma)}{r^{1 / \gamma}(\varsigma) \delta_{0}^{\gamma+1}(\varsigma)}
$$

then (1.1) is oscillatory.

Proof Suppose that there exists a nonoscillatory solution $y$ of (1.1) in $I_{0}$. Without loss of generality, we suppose that $y$ is eventually positive. From Lemma 2.1, we have three cases $(1)-(3)$. Since $y>0$ and $y^{\prime}>0$ in cases (1) and (2), we have that $\lim _{\varsigma \rightarrow \infty} y(\varsigma) \neq 0$.

Now, let case (1) hold. Using Lemma 1.1, we get

$$
y(g(\varsigma, s)) \geq \frac{\lambda_{0} g^{n-1}(\varsigma, s)}{(n-1) ! r^{1 / \gamma}(g(\varsigma, s))} r^{1 / \gamma}(g(\varsigma, s)) y^{(n-1)}(g(\varsigma, s))
$$

for all $\lambda_{0} \in(0,1)$ and sufficiently large $\varsigma$. So, from (3.3), we get that $v(\varsigma)=r(\varsigma)\left(y^{(n-1)}(\varsigma)\right)^{\gamma}>$ 0 is a solution of the delay differential inequality

$$
v^{\prime}(\varsigma)+\left(\frac{\lambda_{0} g^{n-1}(\varsigma, a)}{(n-1) !}\right)^{\gamma} \frac{A[q ; a, b](\varsigma)}{r(g(\varsigma, a))} v(g(\varsigma, a)) \leq 0
$$

From [19, Corollary 1], there exists also a positive solution of (3.1), a contradiction. Assume that case (2) holds. Note that $r(\varsigma)\left(y^{(n-1)}(\varsigma)\right)^{\gamma}$ is nonincreasing, and so

$$
\begin{aligned}
y^{(n-2)}(\nu)-y^{(n-2)}(\varsigma) & =\int_{\varsigma}^{v} \frac{1}{r^{1 / \gamma}(\varrho)}\left(r(\varrho)\left(y^{(n-1)}(\varrho)\right)^{\gamma}\right)^{1 / \gamma} \mathrm{d} \varrho \\
& \leq r^{1 / \gamma}(\varsigma) y^{(n-1)}(\varsigma) \int_{\varsigma}^{v} \frac{1}{r^{1 / \gamma}(\varrho)} \mathrm{d} \varrho .
\end{aligned}
$$

Letting $v \rightarrow \infty$, we get

$$
y^{(n-2)}(\varsigma) \geq-r^{1 / \gamma}(\varsigma) y^{(n-1)}(\varsigma) \delta_{0}(\varsigma)
$$

Next, we define the function $\Theta(\varsigma)$ by

$$
\Theta(\varsigma):=\theta(\varsigma)\left(\frac{r(\varsigma)\left(y^{(n-1)}(\varsigma)\right)^{\gamma}}{\left(y^{(n-2)}(\varsigma)\right)^{\gamma}}+\frac{1}{\delta_{0}^{\gamma}(\varsigma)}\right)
$$

From (3.4), $\Theta(\varsigma)>0$ for $\varsigma \geq \varsigma_{1}$. Therefore, we have

$$
\Theta^{\prime}(\varsigma)=\frac{\theta^{\prime}(\varsigma)}{\theta(\varsigma)} \Theta(\varsigma)+\theta(\varsigma)\left(\frac{\left(r(\varsigma)\left(y^{(n-1)}(\varsigma)\right)^{\gamma}\right)^{\prime}}{\left(y^{(n-2)}(\varsigma)\right)^{\gamma}}-\frac{\gamma r(\varsigma)\left(y^{(n-1)}(\varsigma)\right)^{\gamma+1}}{\left(y^{(n-2)}(\varsigma)\right)^{\gamma+1}}-\frac{\gamma \delta_{0}^{\prime}(\varsigma)}{\delta_{0}^{\gamma+1}(\varsigma)}\right)
$$


it follows from (1.1) and (3.5) that

$$
\begin{aligned}
\Theta^{\prime}(\varsigma) \leq & \frac{\theta^{\prime}(\varsigma)}{\theta(\varsigma)} \Theta(\varsigma)-\theta(\varsigma) \frac{y^{\gamma}(g(\varsigma, a))}{\left(y^{(n-2)}(\varsigma)\right)^{\gamma}} A[q ; a, b](\varsigma) \\
& -\frac{\gamma \theta(\varsigma)}{r^{1 / \gamma}(\varsigma)}\left(\frac{\Theta(\varsigma)}{\theta(\varsigma)}-\frac{1}{\delta_{0}^{\gamma}(\varsigma)}\right)^{(\gamma+1) / \gamma}+\frac{\gamma \theta(\varsigma)}{r^{1 / \gamma}(\varsigma) \delta_{0}^{\gamma+1}(\varsigma)} .
\end{aligned}
$$

Using Lemma 1.1, we get

$$
y(g(\varsigma, a)) \geq \lambda_{1} \frac{g^{n-2}(\varsigma, a)}{n-2 !} y^{(n-2)}(g(\varsigma, a)) .
$$

Thus, (3.6) becomes

$$
\begin{aligned}
\Theta^{\prime}(\varsigma) \leq & \frac{\theta^{\prime}(\varsigma)}{\theta(\varsigma)} \Theta(\varsigma)-\theta(\varsigma)\left(\frac{\lambda_{1} g^{n-2}(\varsigma, a)}{(n-2) !}\right)^{\gamma} A[q ; a, b](\varsigma)+\frac{\gamma \theta(\varsigma)}{r^{1 / \gamma}(\varsigma) \delta_{0}^{\gamma+1}(\varsigma)} \\
& -\frac{\gamma \theta(\varsigma)}{r^{1 / \gamma}(\varsigma)}\left(\frac{\Theta(\varsigma)}{\theta(\varsigma)}-\frac{1}{\delta_{0}^{\gamma}(\varsigma)}\right)^{(\gamma+1) / \gamma} .
\end{aligned}
$$

Using the inequality

$$
A^{(\gamma+1) / \gamma}-(A-B)^{(\gamma+1) / \gamma} \leq \frac{B^{1 / \gamma}}{\gamma}[(1+\gamma) A-B], \quad A B \geq 0,
$$

with $A=\Theta(\varsigma) / \theta(\varsigma), B=1 / \delta_{0}^{\gamma}(\varsigma)$, we obtain

$$
\begin{aligned}
\Theta^{\prime}(\varsigma) \leq & \frac{\theta^{\prime}(\varsigma)}{\theta(\varsigma)} \Theta(\varsigma)-\theta(\varsigma)\left(\frac{\lambda_{1} g^{n-2}(\varsigma, a)}{(n-2) !}\right)^{\gamma} A[q ; a, b](\varsigma)+\frac{\gamma \theta(\varsigma)}{r^{1 / \gamma}(\varsigma) \delta_{0}^{\gamma+1}(\varsigma)} \\
& -\frac{\gamma \theta(\varsigma)}{r^{1 / \gamma}(\varsigma)}\left(\left(\frac{\Theta(\varsigma)}{\theta(\varsigma)}\right)^{(\gamma+1) / \gamma}-\frac{1}{\gamma \delta_{0}(\varsigma)}\left[(1+\gamma) \frac{\Theta(\varsigma)}{\theta(\varsigma)}-\frac{1}{\delta_{0}^{\gamma}(\varsigma)}\right]\right) .
\end{aligned}
$$

Therefore,

$$
\begin{aligned}
\Theta^{\prime}(\varsigma) \leq & \left(\frac{\theta^{\prime}(\varsigma)}{\theta(\varsigma)}+\frac{1+\gamma}{r^{1 / \gamma}(\varsigma) \delta_{0}(\varsigma)}\right) \Theta(\varsigma)-\theta(\varsigma)\left(\frac{\lambda_{1} g^{n-2}(\varsigma, a)}{(n-2) !}\right)^{\gamma} A[q ; a, b](\varsigma) \\
& -\frac{\gamma}{r^{1 / \gamma}(\varsigma) \theta^{1 / \gamma}(\varsigma)} \Theta^{(\gamma+1) / \gamma}(\varsigma)-\frac{\theta(\varsigma)}{r^{1 / \gamma}(\varsigma) \delta_{0}^{\gamma+1}(\varsigma)}+\frac{\gamma \theta(\varsigma)}{r^{1 / \gamma}(\varsigma) \delta_{0}^{\gamma+1}(\varsigma)} .
\end{aligned}
$$

By using the inequality

$$
\nu E-V E^{(\gamma+1) / \gamma} \leq \frac{\gamma^{\gamma}}{(\gamma+1)^{\gamma+1}} \frac{\nu^{\gamma+1}}{V^{\gamma}}, \quad V>0,
$$

with $v=\theta^{\prime}(\varsigma) / \theta(\varsigma)+(1+\gamma) /\left(r^{1 / \gamma}(\varsigma) \delta_{0}(\varsigma)\right), V=\gamma /\left(r^{1 / \gamma}(\varsigma) \theta^{1 / \gamma}(\varsigma)\right)$, and $E=\Theta(\varsigma)$, we find

$$
\begin{aligned}
\Theta^{\prime}(\varsigma) \leq & -\theta(\varsigma)\left(\frac{\lambda_{1} g^{n-2}(\varsigma, a)}{(n-2) !}\right)^{\gamma} A[q ; a, b](\varsigma)+(\gamma-1) \frac{\theta(\varsigma)}{r^{1 / \gamma}(\varsigma) \delta_{0}^{\gamma+1}(\varsigma)} \\
& +\frac{r(\varsigma) \theta(\varsigma)}{(\gamma+1)^{\gamma+1}}\left(\frac{\theta^{\prime}(\varsigma)}{\theta(\varsigma)}+\frac{1+\gamma}{r^{1 / \gamma}(\varsigma) \delta_{0}(\varsigma)}\right)^{\gamma+1} \cdot
\end{aligned}
$$


Integrating this inequality from $\varsigma_{1}$ to $\varsigma$, we find

$$
\int_{\varsigma 1}^{\varsigma}\left(\Phi(\varrho)-\frac{r(\varrho) \theta(\varrho)}{(\gamma+1)^{\gamma+1}}\left(\frac{\theta^{\prime}(\varrho)}{\theta(\varrho)}+\frac{1+\gamma}{r^{1 / \gamma}(\varrho) \delta_{0}(\varrho)}\right)^{\gamma+1}\right) \mathrm{d} \varrho \leq \Theta\left(\varsigma_{1}\right)
$$

which contradicts (3.2).

Next, using Theorem 2.5, it follows from $(\Omega)$ and (2.14) that $y \notin \mathbf{K}$, and so $y$ does not satisfy case (3).

This completes the proof.

Corollary 3.1 Assume that $(\Omega)$ and (2.1) hold and there exist functions $\rho, \theta \in C^{1}\left(I_{0},(0, \infty)\right)$ such that (2.14) and (3.2) hold. If

$$
\liminf _{\zeta \rightarrow \infty} \int_{g(\zeta, a)}^{\zeta} \frac{\left(\lambda_{0} g^{n-1}(\xi, a)\right)^{\gamma}}{((n-1) !)^{\gamma} r(g(\xi, a))} A[q ; a, b](\xi) \mathrm{d} \xi>\frac{1}{\mathrm{e}}
$$

then (1.1) is oscillatory.

Proof Applying a well-known criterion [7, Theorem 2] for first-order equation (3.1) to be oscillatory, we obtain immediately criterion (3.7).

Theorem 3.2 Assume that $n=4,(\Omega)$ and (2.1) hold. If there exist functions $\varphi, \phi, \rho \in$ $C^{1}\left(I_{0},(0, \infty)\right)$ such that

$$
\begin{aligned}
& \int_{\varsigma_{0}}^{\infty}\left(\varphi(s)\left(\frac{g(s, a)}{s}\right)^{3 \gamma} A[q ; a, b](s)-\frac{2^{\gamma}}{(\gamma+1)^{\gamma+1}} \frac{r(s)\left(\varphi^{\prime}(s)\right)^{\gamma+1}}{\left(\mu s^{2} \varphi(s)\right)^{\gamma}}\right) \mathrm{d} s=\infty, \\
& \int_{\varsigma_{0}}^{\infty}\left(\phi(\xi) \int_{\xi}^{\infty}\left(\frac{1}{r(v)} \int_{\nu}^{\infty} \frac{g^{\gamma}(s, a)}{s^{\gamma}} A[q ; a, b](s) \mathrm{d} s\right)^{1 / \gamma} \mathrm{d} v-\frac{\left(\phi^{\prime}(\xi)\right)^{2}}{4 \phi(\xi)}\right) \mathrm{d} \xi \\
& \quad=\infty, \\
& \lim _{\varsigma \rightarrow \infty} \sup \frac{\delta_{0}^{\gamma}(\varsigma)}{\rho(\varsigma)} \int_{\varsigma_{0}}^{\varsigma}\left(\rho(\varrho)\left(\frac{\lambda}{2 !} g^{2}(\varrho, a)\right)^{\gamma} A[q ; a, b](\varrho)-\frac{r(\varrho)\left(\rho^{\prime}(\varrho)\right)^{\gamma+1}}{(\gamma+1)^{(\gamma+1)} \rho^{\gamma}(\varrho)}\right) \mathrm{d} \varrho \\
& >1,
\end{aligned}
$$

and

$$
\limsup _{\zeta \rightarrow \infty} \frac{\delta_{2}^{\gamma}(\varsigma)}{\rho(\varsigma)} \int_{\varsigma 1}^{\varsigma}\left(\rho(\zeta) h^{\gamma \eta} A[q ; a, b](\zeta)-\frac{\rho^{-\gamma}(\zeta)\left(\rho^{\prime}(\zeta)\right)^{\gamma+1}}{(\gamma+1)^{(\gamma+1)} \delta_{1}^{\gamma}(\zeta)}\right) \mathrm{d} \zeta>1
$$

for some $\lambda, \mu \in(0,1)$, then (1.1) is oscillatory.

Proof Suppose that there exists a nonoscillatory solution $y$ of (1.1) in $I_{0}$. Without loss of generality, we suppose that $y$ is eventually positive. Using [1, Lemma 2.2.1], there exist four possible cases:

$$
\begin{aligned}
& \text { C1: } \quad y^{\prime}(\varsigma)>0, \quad y^{\prime \prime}(\varsigma)>0 \quad \text { and } \quad y^{\prime \prime \prime}(\varsigma)>0 \text {; } \\
& \mathrm{C} 2: \quad y^{\prime}(\varsigma)>0, \quad y^{\prime \prime}(\varsigma)<0 \quad \text { and } \quad y^{\prime \prime \prime}(\varsigma)>0 \text {; }
\end{aligned}
$$


C3: $\quad y^{\prime}(\varsigma)>0, \quad y^{\prime \prime}(\varsigma)>0 \quad$ and $\quad y^{\prime \prime \prime}(\varsigma)<0$;

C4: $\quad y^{\prime}(\varsigma)<0, \quad y^{\prime \prime}(\varsigma)>0$ and $y^{\prime \prime \prime}(\varsigma)<0$.

The proof of the case where $\mathrm{C} 1$ or $\mathrm{C} 2$ holds is the same as that of [16, Theorem 2.1].

Assume that C3 holds. Proceeding as in the proof of Theorem 3.1, we obtain that (3.6) holds. Thus, we get

$$
\begin{aligned}
\Theta^{\prime}(\varsigma) \leq & \frac{\rho^{\prime}(\varsigma)}{\rho(\varsigma)} \Theta(\varsigma)-\frac{\rho(\varsigma) y^{\gamma}(g(\varsigma, a))}{\left(y^{\prime \prime}(\varsigma)\right)^{\gamma}} A[q ; a, b](\varsigma) \\
& -\frac{\gamma}{r^{1 / \gamma}(\varsigma) \rho^{1 / \gamma}(\varsigma)}\left(\Theta(\varsigma)-\frac{\rho(\varsigma)}{\delta_{0}^{\gamma}(\varsigma)}\right)^{(\gamma+1) / \gamma}-\frac{\gamma \rho(\varsigma) \delta_{0}^{\prime}(\varsigma)}{\delta_{0}^{\gamma+1}(\varsigma)} .
\end{aligned}
$$

Using Lemma 1.2 with $D=\rho^{\prime}(\varsigma) / \rho(\varsigma), M=\gamma /\left(r^{1 / \gamma}(\varsigma) \rho^{1 / \gamma}(\varsigma)\right), N=\rho(\varsigma) / \delta_{0}^{\gamma}(\varsigma)$, and $\xi=\Theta$, we obtain

$$
\begin{aligned}
\Theta^{\prime}(\varsigma) \leq & -\frac{\rho(\varsigma) y^{\gamma}(g(\varsigma, a))}{\left(y^{\prime \prime}(\varsigma)\right)^{\gamma}} A[q ; a, b](\varsigma) \\
& -\frac{\gamma \rho(\varsigma) \delta_{0}^{\prime}(\varsigma)}{\delta_{0}^{\gamma+1}(\varsigma)}+\frac{\rho^{\prime}(\varsigma)}{\delta_{0}^{\gamma}(\varsigma)}+\frac{r(\varsigma)\left(\rho^{\prime}(\varsigma)\right)^{\gamma+1}}{(\gamma+1)^{\gamma+1} \rho^{\gamma}(\varsigma)}
\end{aligned}
$$

or

$$
\Theta^{\prime}(\varsigma) \leq-\frac{\rho(\varsigma) y^{\gamma}(g(\varsigma, a))}{\left(y^{\prime \prime}(\varsigma)\right)^{\gamma}} A[q ; a, b](\varsigma)+\left(\frac{\rho(\varsigma)}{\delta_{0}^{\gamma}(\varsigma)}\right)^{\prime}+\frac{r(\varsigma)\left(\rho^{\prime}(\varsigma)\right)^{\gamma+1}}{(\gamma+1)^{\gamma+1} \rho^{\gamma}(\varsigma)} .
$$

From Lemma 1.1, we have

$$
\Theta^{\prime}(\varsigma) \leq-\rho(\varsigma)\left(\frac{\lambda}{2 !} g^{2}(\varsigma, a)\right)^{\gamma} A[q ; a, b](\varsigma)+\left(\frac{\rho(\varsigma)}{\delta_{0}^{\gamma}(\varsigma)}\right)^{\prime}+\frac{r(\varsigma)\left(\rho^{\prime}(\varsigma)\right)^{\gamma+1}}{(\gamma+1)^{\gamma+1} \rho^{\gamma}(\varsigma)} .
$$

Integrating the above inequality from $\varsigma_{1}$ to $\varsigma$, we find

$$
\begin{aligned}
\Theta(\varsigma)-\Theta\left(\varsigma_{1}\right) \leq & -\int_{\varsigma 1}^{\varsigma}\left(\rho(\varrho)\left(\frac{\lambda}{2 !} g^{2}(\varrho, a)\right)^{\gamma} A[q ; a, b](s)-\frac{r(\varrho)\left(\rho^{\prime}(\varrho)\right)^{\gamma+1}}{(\gamma+1)^{(\gamma+1)} \rho^{\gamma}(\varrho)}\right) \mathrm{d} \varrho \\
& +\frac{\rho(\varsigma)}{\delta_{0}^{\gamma}(\varsigma)}-\frac{\rho\left(\varsigma_{1}\right)}{\delta_{0}^{\gamma}\left(\varsigma_{1}\right)} .
\end{aligned}
$$

From the definition of $\Theta$, we see that

$$
\begin{aligned}
& \int_{\varsigma 1}^{\varsigma}\left(\rho(\varrho)\left(\frac{\lambda}{2 !} g^{2}(\varrho, a)\right)^{\gamma} A[q ; a, b](s)-\frac{r(\varrho)\left(\rho^{\prime}(\varrho)\right)^{\gamma+1}}{(\gamma+1)^{(\gamma+1)} \rho^{\gamma}(\varrho)}\right) \mathrm{d} \varrho \\
& \quad \leq-\frac{\rho(\varsigma) r(\varsigma)\left(y^{\prime \prime \prime}(\varsigma)\right)^{\gamma}}{\left(y^{\prime \prime}(\varsigma)\right)^{\gamma}}+\frac{\rho\left(\varsigma_{1}\right) r\left(\varsigma_{1}\right)\left(y^{\prime \prime \prime}\left(\varsigma_{1}\right)\right)^{\gamma}}{\left(y^{\prime \prime}\left(\varsigma_{1}\right)\right)^{\gamma}} .
\end{aligned}
$$

This provides

$$
\int_{\varsigma 1}^{\varsigma}\left(\rho(\varrho)\left(\frac{\lambda}{2 !} g^{2}(\varrho, a)\right)^{\gamma} A[q ; a, b](s)-\frac{r(\varrho)\left(\rho^{\prime}(\varrho)\right)^{\gamma+1}}{(\gamma+1)^{(\gamma+1)} \rho^{\gamma}(\varrho)}\right) \mathrm{d} \varrho \leq \frac{\rho(\varsigma)}{\delta_{0}^{\gamma}(\varsigma)} .
$$


Hence,

$$
\frac{\delta_{0}^{\gamma}(\varsigma)}{\rho(\varsigma)} \int_{\varsigma 1}^{\varsigma}\left(\rho(\varrho)\left(\frac{\lambda}{2 !} g^{2}(\varrho, a)\right)^{\gamma} A[q ; a, b](s)-\frac{r(\varrho)\left(\rho^{\prime}(\varrho)\right)^{\gamma+1}}{(\gamma+1)^{(\gamma+1)} \rho^{\gamma}(\varrho)}\right) \mathrm{d} \varrho \leq 1,
$$

which contradicts (3.10).

Next, using Theorem 2.5 with $n=4$, it follows from $(\Omega)$ and (3.11) that $y \notin \mathbf{K}$, and so $y$ does not satisfy case C4.

This completes the proof.

\section{Examples}

Example 4.1 Consider the fourth-order DE

$$
\left(\mathrm{e}^{\gamma \varsigma}\left(y^{\prime \prime \prime}(\varsigma)\right)^{\gamma}\right)^{\prime}+q_{0} \mathrm{e}^{\gamma \varsigma} A[y \circ g ; \lambda, 1](\varsigma)=0
$$

where $\varsigma \geq 1, \lambda \in(0,1-1 / \mathrm{e}), g(\varsigma, s)=s \varsigma$, and $q_{0}>0$. Then we get $\delta_{m}(\varsigma)=\mathrm{e}^{-\varsigma}$ for $m=0,1,2$. Moreover, it is easy to verify that conditions (2.1), (3.2), and (3.7) are satisfied.

By using the fact that $\mathrm{e}^{v}>\mathrm{e} v$ for $v>0$, we get

$$
\frac{\delta_{2}(g(\varsigma, s))}{\delta_{2}(\varsigma)} \geq \frac{\delta_{2}(g(\varsigma, a))}{\delta_{2}(\varsigma)}=\mathrm{e}^{(1-\lambda) \varsigma}>\mathrm{e}(1-\lambda) \varsigma \geq \mathrm{e}(1-\lambda):=h>1 .
$$

From Theorems 2.2 and 2.3, equation (4.1) has no Kneser solutions if

$$
\eta=\left(\frac{q_{0}}{\gamma}\right)^{1 / \gamma}(1-\lambda)^{1 / \gamma}>1
$$

or

$$
h^{\eta} \eta=(\mathrm{e}(1-\lambda))^{(1-\lambda)^{1 / \gamma}\left(\frac{q_{0}}{\gamma}\right)^{1 / \gamma}}\left(\frac{q_{0}}{\gamma}\right)^{1 / \gamma}(1-\lambda)^{1 / \gamma}>1
$$

holds.

Next, condition (2.14) takes the form

$$
(\mathrm{e}(1-\lambda))^{\gamma(1-\lambda)^{1 / \gamma}\left(\frac{q_{0}}{\gamma}\right)^{1 / \gamma}} q_{0}(1-\lambda)>\left(\frac{\gamma}{\gamma+1}\right)^{\gamma+1}
$$

By Corollary 3.1, equation (4.1) is oscillatory provided that (4.2) holds.

Example 4.2 Consider the fourth-order DE

$$
\left(\varsigma^{5} y^{\prime \prime \prime}(\varsigma)\right)^{\prime}+q_{0} \varsigma A\left[y \circ g ; \lambda_{2}, 1\right](\varsigma)=0
$$

where $\varsigma \geq 1, \lambda_{2} \in(0,1), g(\varsigma, s)=s \varsigma$, and $q_{0}>0$. Then we have that $\delta_{0}(\varsigma)=1 / 4 \varsigma^{4}, \delta_{1}(\varsigma)=$ $1 / 12 \varsigma^{3}$, and $\delta_{2}(\varsigma)=1 / 24 \varsigma^{2}$. Moreover, it is easy to verify that conditions (3.8) and (3.9) are satisfied. Using Theorem 3.2, equation (4.3) is oscillatory if

$$
(3.10) \rightarrow \frac{\lambda_{1}}{8} \lambda_{2}^{2} q_{0}\left(1-\lambda_{2}\right)>1
$$


and

$$
(3.11) \rightarrow \frac{1}{24}\left(\frac{1}{\lambda_{2}}\right)^{\frac{q_{0}}{48}\left(1-\lambda_{2}\right)} q_{0}\left(1-\lambda_{2}\right)>\frac{1}{2}
$$

hold.

Remark 4.1 Consider the fourth-order DE (4.3). Condition (3.7) is not satisfied, so Theorem 3.1 cannot be applied. Thus, Theorem 3.2 provides an applicable criterion when Theorem 3.1 fails to apply.

\section{Acknowledgements}

The authors are grateful to the editors and two anonymous referees for a very thorough reading of the manuscript and for pointing out some inaccuracies. The second author would like to extend his sincere appreciation to the Deanship of Scientific Research, King Saud University for its funding through Research Unit of Common First Year Deanship.

\section{Funding}

Not applicable.

\section{Availability of data and materials}

No data sharing (where no datasets are produced).

\section{Competing interests}

The authors declare that they have no competing interests.

\section{Authors' contributions}

All authors contributed equally to this work. All authors read and approved the final manuscript.

\section{Author details}

${ }^{1}$ Department of Mathematics, Faculty of Science, Mansoura University, 35516 Mansoura, Egypt. ${ }^{2}$ Department of Mathematics, Faculty of Education-Al-Nadirah, Ibb University, Ibb, Yemen. ${ }^{3}$ Department of Basic Sciences, Common First Year, King Saud University, Riyadh 11451, Saudi Arabia.

\section{Publisher's Note}

Springer Nature remains neutral with regard to jurisdictional claims in published maps and institutional affiliations.

Received: 11 August 2020 Accepted: 5 May 2021 Published online: 13 May 2021

\section{References}

1. Agarwal, R.P., Grace, S.R., O'Regan, D.: Oscillation Theory for Difference and Functional Differential Equations. Dekker, Dordrecht (2000)

2. Baculikova, B., Dzurina, J., Graef, J.R.: On the oscillation of higher-order delay differential equations. J. Math. Sci. 187(4), 387-400 (2012)

3. Chatzarakis, G.E., Elabbasy, E.M., Moaaz, O., Mahjoub, H.: Global analysis and the periodic character of a class of difference equations. Axioms 8(4), 131 (2020)

4. Graef, J.R., Grace, S.R., Tunc, E.: Oscillation of even-order advanced functional differential equations. Publ. Math. (Debr.) 93(3-4), 445-455 (2018)

5. Graef, J.R., Grace, S.R., Tunc, E.: Oscillatory behavior of even-order nonlinear differential equations with a sublinear neutral term. Opusc. Math. 39(1), 39-47 (2019)

6. Kiguradze, I., Chanturia, T.: Asymptotic Properties of Solutions of Nonautonomous Ordinary Differential Equations. Mathematics and Its Applications (Soviet Series), vol. 89. Kluwer Academic, Dordrecht (1993) Translated from the 1985 Russian original

7. Kitamura, Y., Kusano, T.: Oscillation of first-order nonlinear differential equations with deviating arguments. Proc. Am. Math. Soc. 78(1), 64-68 (1980)

8. Li, T., Rogovchenko, Y.V.: Asymptotic behavior of higher-order quasilinear neutral differential equations. Abstr. Appl. Anal. 2014, 1 (2014)

9. Li, T., Rogovchenko, Y.V.: On asymptotic behavior of solutions to higher-order sublinear Emden-Fowler delay differential equations. Appl. Math. Lett. 67, 53-59 (2017)

10. Li, T., Rogovchenko, Y.V.: On the asymptotic behavior of solutions to a class of third-order nonlinear neutral differential equations. Appl. Math. Lett. 105, 106293 (2020)

11. Moaaz, O.: Oscillatory behavior of solutions of odd-order nonlinear delay differential equations. Adv. Differ. Equ. 2020 $357(2020)$

12. Moaaz, O., Anis, M., Baleanu, D., Muhib, A.: More effective criteria for oscillation of second-order differential equations with neutral arguments. Mathematics 8(6), 986 (2020) 
13. Moaaz, O., Baleanu, D., Muhib, A.: New aspects for non-existence of Kneser solutions of neutral differential equations with odd-order. Mathematics 8(4), 494 (2020)

14. Moaaz, O., Dassios, I., Bazighifan, O., Muhib, A.: Oscillation theorems for nonlinear differential equations of fourth-order. Mathematics 8, 520 (2020)

15. Moaaz, O., Dassios, I., Muhsin, W., Muhib, A.: Oscillation Theory for Non-linear Neutral Delay Differential Equations of Third Order, vol. 10, p. 4855. Appl. Sci., Braking (2020)

16. Moaaz, O., Elabbasy, E.M., Bazighifan, O.: On the asymptotic behavior of fourth-order functional differential equations. Adv. Differ. Equ. 2017, 261 (2017)

17. Moaaz, O., Muhib, A.: New oscillation criteria for nonlinear delay differential equations of fourth-order. Appl. Math. Comput. 377, $125192(2020)$

18. Ou, C.H., Wong, J.S.W.: Oscillation and non-oscillation theorems for superlinear Emden-Fowler equations of the fourth order. Ann. Mat. Pura Appl. 183, 25-43 (2004)

19. Wong, J.S.W.: On the generalized Emden-Fowler equation. SIAM Rev. 17, 339-360 (1975)

20. Zhang, C., Agarwal, R.P., Bohner, M., Li, T.: New results for oscillatory behavior of even-order half-linear delay differential equations. Appl. Math. Lett. 26, 179-183 (2013)

21. Zhang, C., Li, T., Saker, S.H.: Oscillation of fourth order delay differential equations. J. Math. Sci. 201(3) (2014)

22. Zhang, C., Li, T., Sun, B., Thandapani, E.: On the oscillation of higher-order half-linear delay differential equations. Appl. Math. Lett. 24, 1618-1621 (2011)

\section{Submit your manuscript to a SpringerOpen ${ }^{\circ}$ journal and benefit from:}

- Convenient online submission

- Rigorous peer review

- Open access: articles freely available online

- High visibility within the field

- Retaining the copyright to your article

Submit your next manuscript at $\boldsymbol{\nabla}$ springeropen.com 